\title{
Using Python with Smoke and JWST Mirrors
}

\author{
Warren J. Hack ${ }^{\ddagger *}$, Perry Greenfield ${ }^{\ddagger}$, Babak Saif ${ }^{\ddagger}$, Bente Eegholm ${ }^{\ddagger}$
}

Abstract-We will describe how the Space Telescope Science Institute is using Python in support of the next large space telescope, the James Webb Space Telescope (JWST). We will briefly describe the 6.5 meter segmented-mirror infra-red telescope, currently planned for a 2014 launch, and its science goals. Our experience with Python has already been employed to study the variation of the mirror and instrument support structures during cyrogenic cool-down from ambient temperatures to 30 Kelvin with accuracies better than 10 nanometers using a speckle interferometer. Python was used to monitor, process (initially in near real-time) and analyze over 15 TB of data collected. We are currently planning a metrology test that will collect 10 TB of data in 7 minutes. We will discuss the advantages of using Python for each of these projects.

Index Terms - astronomy, telescope, NASA, measure, real-time, big data

\section{Introduction}

The James Webb Space Telescope (JWST) will be NASA's next Great Observatory. It will be an infrared-optimized telescope with a $6.5 \mathrm{~m}$ primary mirror made up of 18 -separate segments which will be launched no sooner than 2015 by an Ariane 5 into an orbit at the second Langragian (L2) point. This orbital position, about 1.5 million $\mathrm{km}$ from the Earth, keeps the Sun behind the Earth at all times making it easier to shield the telescope and keep it cool. The Hubble Space Telescope (HST), by comparison, is a telescope in a $570 \mathrm{~km}$ high orbit with a solid $2.4 \mathrm{~m}$ primary mirror optimized for UV and optical observations. A lot of effort will go into building and testing JWST, as it did with HST, to get it to work as desired and as reliably as possible once launched. However, unlike HST, there will not be any possibility of performing a repair mission. The primary structure of JWST will be made of carbonfiber composites in order to be lightweight enough for launch while still providing the necessary strength and rigidity to support such a large set of mirrors and instrument packages. The primary mirror itself will be composed of 18 separate hexagonal segments. These segments will be mounted onto a backplane with actuators that will allow the segments to be aligned to match one common optical surface that represents a single mirror with a diameter of $6.5 \mathrm{~m}$.

A test article, the Backplane Stability Test Article (BSTA), was manufactured using the same materials, techniques, and design principles being developed for constructing the entire telescope. Extensive thermal-vacuum chamber testing was conducted on the

* Corresponding author: hack@stsci.edu

¥ Space Telescope Science Institute

Copyright $@ 2010$ Warren J. Hack et al. This is an open-access article distributed under the terms of the Creative Commons Attribution License, which permits unrestricted use, distribution, and reproduction in any medium, provided the original author and source are credited.

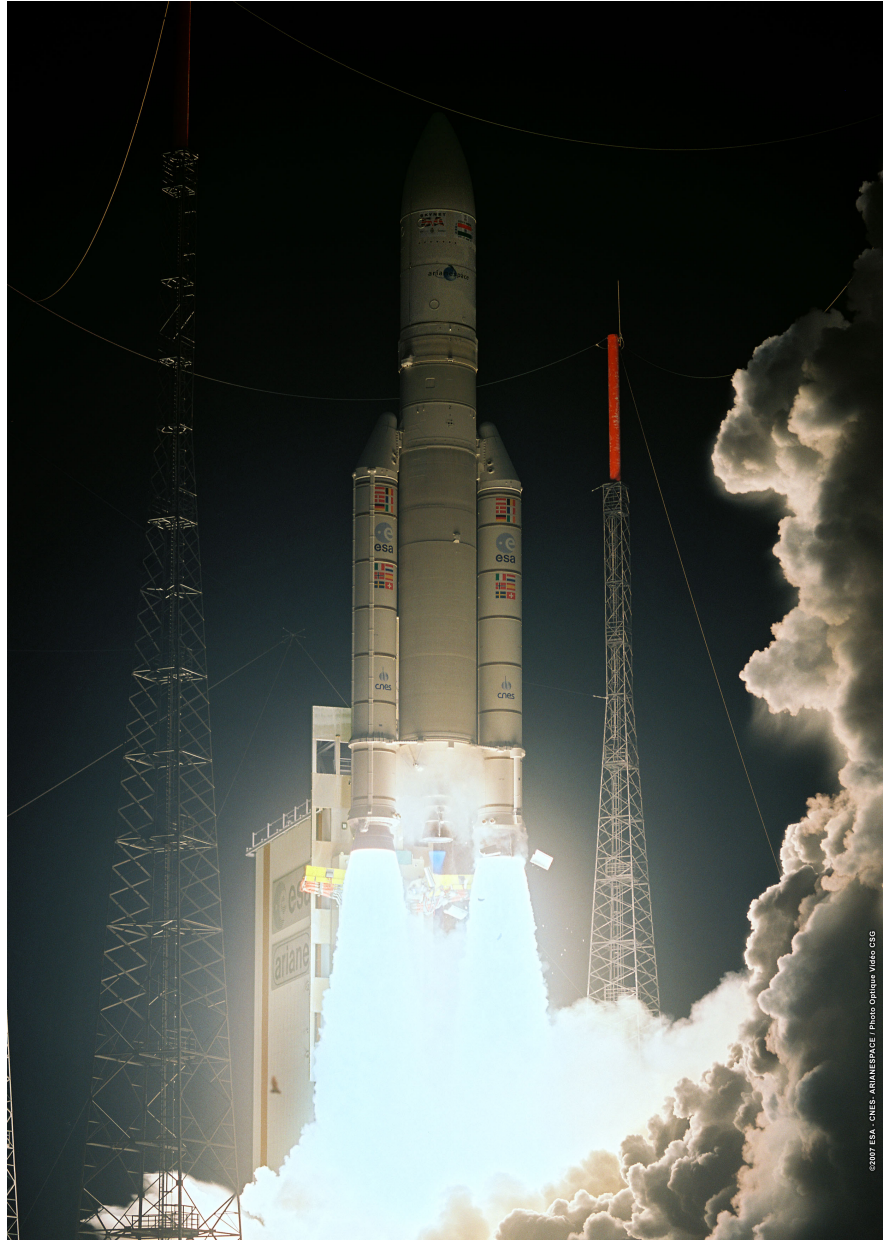

Fig. 1: An Ariane 5 launch similar to the launcher that will be used to place JWST into orbit about the L2 point, the orbital position that keeps the Sun behind the Earth all the time as viewed from JWST. Photo: ESA.

BSTA to verify that it will meet the stringent requirements necessary for JWST to work; specifically, that it will remain stable to within $38 \mathrm{~nm}$ over the orbital operational temperature range of 30$60 \mathrm{~K}$. These tests required the development of specialized software to collect and process all the necessary data. Such software was written in Python and is described in the following sections.

\section{Testing the BSTA}

NASA required a test that demonstrated the stability of this engineering article and which verified that the design and construction 


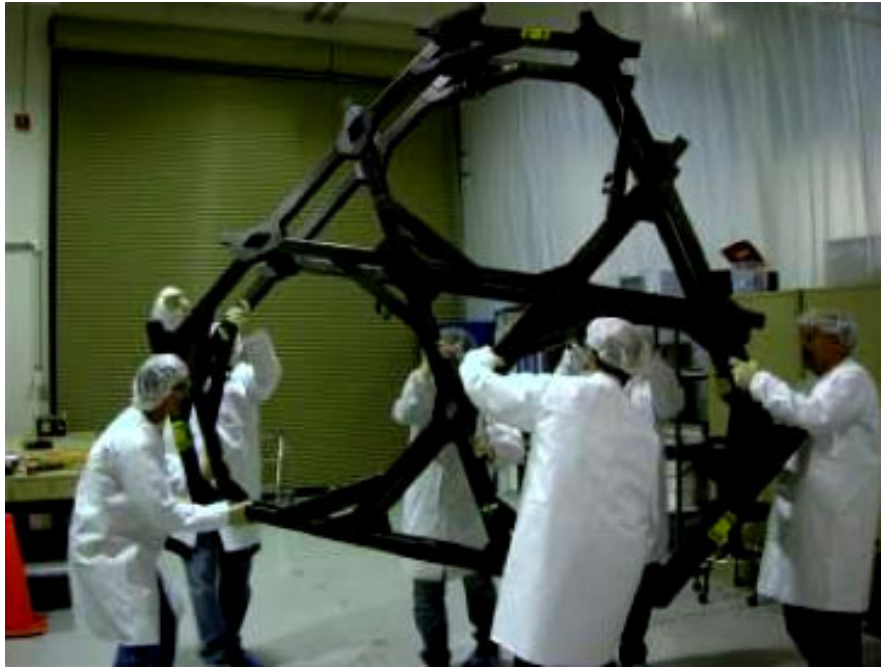

Fig. 2: Engineers move the Backplane Stability Test Article (BSTA) into position for the thermal vacuum test. This test piece represents a section of the backplane that will support only 3 of the 18 mirror segments of the primary mirror for JWST.

techniques will work to meet the requirements of the telescope: namely, that it will remain stable to within $68 \mathrm{~nm}$ from $30-50 \mathrm{~K}$ $\left(-405\right.$ to $\left.-370^{\circ} \mathrm{F}\right)$. They also wished to track the structural changes from ambient $(\sim 295 \mathrm{~K})$ to cryogenic temperatures $(\sim 30 \mathrm{~K})$ in order to better understand the accuracy of their structural models. The primary test equipment, a special interferometer, did have software to take measurements, but that software was not suited for the needs of this test. This required the development of specialized software to support the test.

Normal interferometry is used with optical elements where the reflected or transmitted laser signal remains spatially coherent over large areas. Speckle interferometry is intended to be used with non- optical surfaces, that is, surfaces that are optically rough on very small spatial scales. When illuminated by a laser, such surfaces typically show "speckles" that result from random points where the reflections from the surface are relatively coherent (as compared to the darker areas where the reflections mostly cancel out through interference). While the phase of speckles varies randomly from one spot on the article to the next, and thus cannot be used for any spatial comparison from a single image, how the phase for a specific speckle changes, does indicate how the surface is moving relative to the laser; in this way speckle interferometers can be used to determine how surfaces are changing in time. The BSTA, although intended to hold JWST mirror segments, has no optical surfaces of its own. In order to understand how the structure changed with temperature it was necessary to use the Electronic Speckle Pattern Interferometer (ESPI).

The ESPI laser illuminates the surface of the BSTA, then recombines the reflected signal with a reference beam split from the same laser pulse, to create an interferometric map of the surface speckles. As the structure moves, the speckles change phase reflecting the change in interference between the incident and reference laser pulses. However, those phases cycle from $-\pi$ to $\pi$ and back as the surface continues to move. This required the use of phase unwrapping across the surface, spatial phase unwrapping, using an algorithm developed by the manufacturers of the ESPI.

As the surface tilted during the test, it produced fringes where

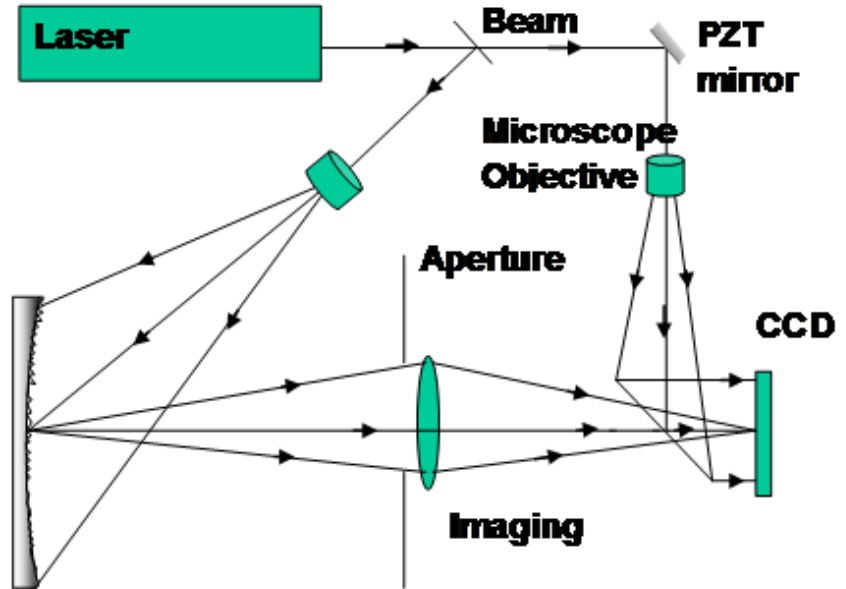

Back plane

Fig. 3: Schematic of ESPI showing how the ESPI measures the change in the object due to thermal or mechanical stress by tracking the speckles' phase change on the surface.

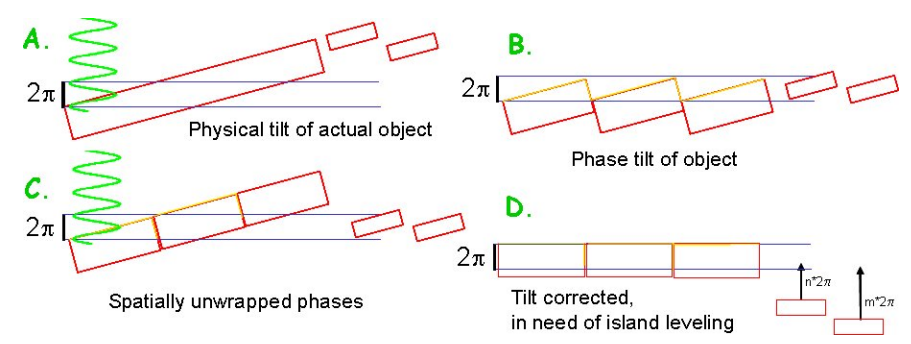

Fig. 4: Schematic showing how bulk tilt introduces multiple $2 \pi$ variations across the structure and how it gets corrected in processing, allowing for relative variations to be measured across the surface as described in "Testing the BSTA".

the phases across the structure would transition from $\pi$ to $-\pi$. This tilt needed to be removed in order to allow us to measure the relative changes from one region of the structure to another.

Since the measured phase is ambiguous by multiples of $2 \pi$, special techniques are required to remove these ambiguities. One is to presume that continuous surfaces have continuous phase, and any discontinuities on continuous surfaces are due to phase wrapping. Thus such discontinuities can be "unwrapped" to produce spatially continuous phase variations. Another presumption is that even though the general position and tilt of the entire structure may change greatly from one exposure to another, the relative phase shape of the structure will not change rapidly in time once bulk tilt and displacement are removed.

The following figures show the consequent phase wraps when a surface has any significant tilt. One can perform spatial phase unwrapping on spatially contiguous sections. Gross tilts are fit to the largest contiguous sections, and then the average tilt is removed (as well as the average displacement). However, there are areas of interest (the mirror pad supports) which are discontiguous and as a result possibly several factors of $2 \pi$ offset in reality as a result of the tilt, and thus improperly corrected when tilts are removed. Since these areas are assumed to change slowly in time, temporal phase unwrapping is applied to these areas.

The entire ESPI system, hardware and software, was built by 4D Technologies under the guidance of one of our team members, 


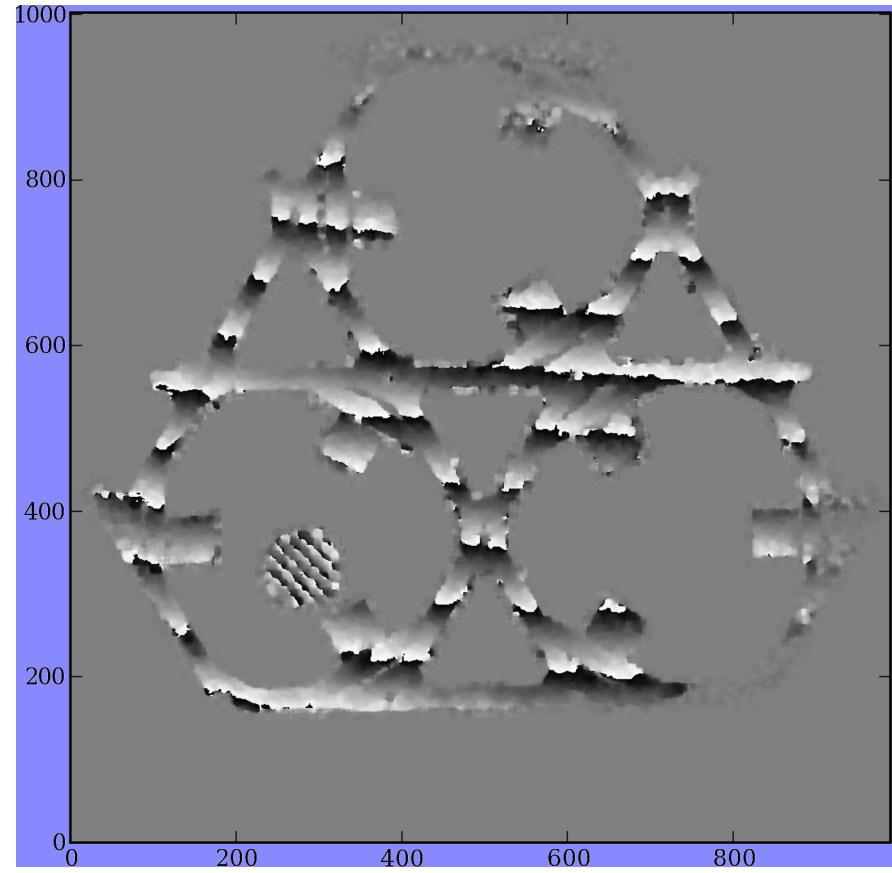

Fig. 5: A sample ESPI image illustrating the fringes that build up due to bulk tilts. These fringes get "unwrapped" to produce spatially contiguous phase variations across the surface of the object.

Babak. The commercial software from 4D Technologies that came with the ESPI hardware had algorithms for performing the spatial unwrapping using a GUI interface for interactive operation. This interface, though, was unable to support the needs of the test; namely, that it would need to continuously take 5 images/second for 24 hours/day for up to 6 weeks at a time. Thus, we needed to write our own specialized software to support the test.

\section{Python to the Rescue}

Many of the requirements for any software that needed to be written were unknowable, not just unknown, for a number of reasons. No test had ever been conducted like this before, so there was no experience to draw upon to foresee what problems may arise during the test. Concerns ranged from whether the laser output could be maintained at a stable level over such a long period of time given that the output was dependent on the ambient temperature of the test facility. This drove the requirement to monitor in near-real-time the laser intensity as measured from the observations themselves. These results were compared with occasional checks of the laser output using burn paper in the laser path, creating a bit of smoke in the process, to insure that the monitoring was accurately tracking the health of the laser.

We also had no certainty about what phase-unwrapping algorithms were going to work until the test actually started. Test conditions such as residual vibrations in the test rig could seriously impact our ability to measure the surface changes we were after and potentially require changes to how the phase-unwrapping algorithms needed to be applied. It was only after the test started that these effects would be known, requiring the ability to update the data acquisition and processing code on the fly to accommodate the quality of the test data.

Finally, the code had to be easily adaptable and capable of handling massive amounts of data in as close to real time as possible! Python offered the best possible choice for addressing

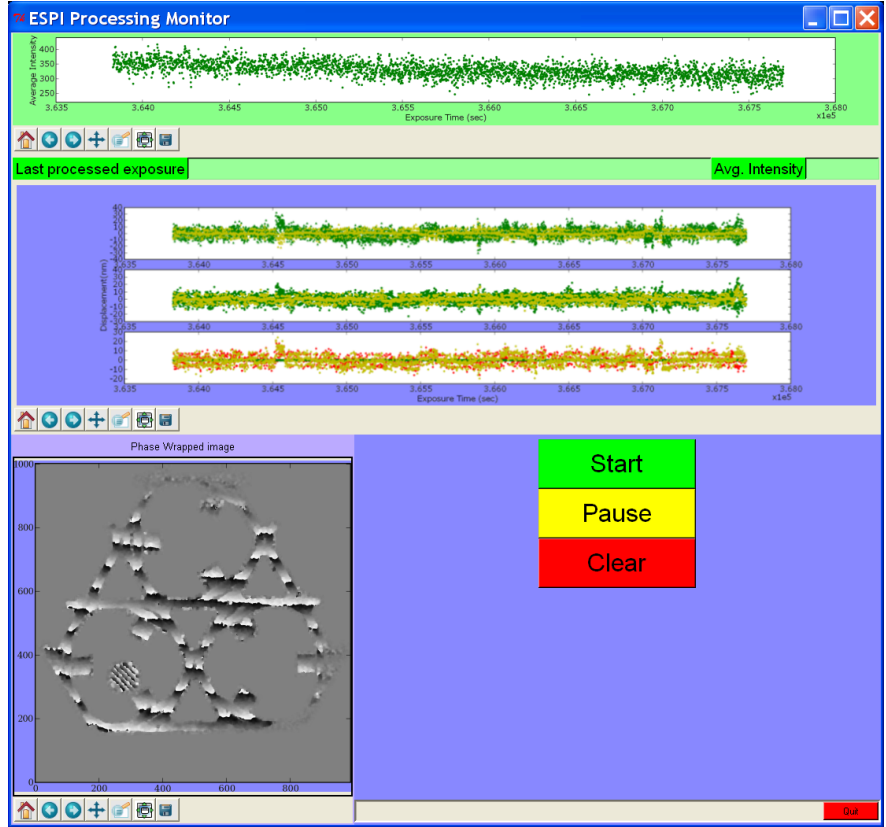

Fig. 6: This snapshot of the ESPI Monitoring GUI in operation illustrates the near-real-time monitoring plots and image display used to track the health of the laser and quality of the data and subsequent processing.

these challenges in supporting this test. It allowed us to develop code rapidly to adjust for the test conditions during the test with minimal impact. The plotting and array-handling libraries, specifically matplotlib and numpy, proved robust and fast enough to keep up with the near-real-time operations. The commercial software that came with ESPI hardware had also been written in Python and $\mathrm{C}$, so Python allowed us to interface to that code to run our own custom processing code using the commercial algorithms for data acquisition and phase-unwrapping.

Our data acquisition system used custom code to automate the operation of the commercial software used to interface with the ESPI camera. This module was run under the commercial software's own Python environment in order to most easily access their camera's API and stored the images in real time on a storage server. The remainder of the processing required the use of the Python API to the commercial software's functions to perform the phase unwrapping. As a result of this extended processing, the remainder of the code could only process and monitor the results of every 5th image taken during the test. This monitoring was performed using a custom Tkinter GUI which provided plots of a couple of key processing results, and an image display of the latest processed image, all using matplotlib.

This data processing pipeline was set up using 4 PCs and a $15 \mathrm{~Tb}$ storage server. A separate PC was dedicated to each of the processing steps; namely, data acquisition, initial phase unwrapping, measuring of regions, and monitoring of the processing. This distributed system was required in order to support the data acquisition rate for the test: $51004 \times 996$ pixel images per second for 24 hours a day for 6 uninterrupted weeks. A total of approximately $11 \mathrm{~Tb}$ of raw data was eventually acquired during the test. These raw observations were later reprocessed several times using the original set of 4 PCs from the test as well as additional PCs all running simultaneously to refine the results in much less than real time using all the lessons learned while the test 


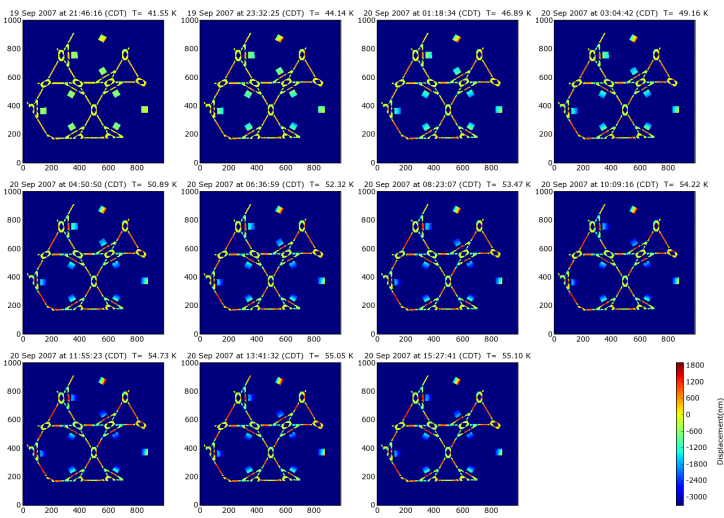

Fig. 7: Mosaic of sample processed measurements of the BSTA as the temperature changed from $40 \mathrm{~K}$ to $60 \mathrm{~K}$, matching the operational temperature range of JWST. This mosaic illustrates how the structure was measured to change as the temperature changed.

was in progress. This reprocessing effort represented the simplest possible case of parallel processing, where separate sets of data could be processed independently on separate systems. No other use of parallel processing techniques was implemented for the test or subsequent reprocessing.

\section{Results}

BSTA data analysis measured the slope of the data, expansion due to temperature, with an RMS of $25.2 \mathrm{~nm} / \mathrm{K}$, well within the $36.8 \mathrm{~nm} / \mathrm{K}$ requirement for meeting NASA's goals. These measurements were based on calibrations which had RMS values less than $5 \mathrm{~nm}$ around the measured slope.

Python allowed for rapid development of a near-real-time processing pipeline spread across multiple systems which we were able to revise quickly as needed during the test. The fact that the commercial software was written using Python also allowed us to interface with it to use their C-based algorithms for data acquisition and phase-unwrapping. Equally importantly, we were able to implement changes in the processing algorithms while the test was underway to address aspects of the data quality that were not expected when the test began. This software, though, can not be distributed as it was designed explicitly to support the JWST tests alone. The success of this test, though, resulted in establishing the ESPI as a resource for later tests, and this software will be used as the framework for supporting additional tests of JWST in the coming years.

\section{Future Tests}

The development of the software for the ESPI tests validated its utility to measure the shape of structures to nanometer accuracies. Additional testing of the actual structure built for use in supporting all 18 segments of the primary mirror for JWST will require this level of accuracy, albeit under very different testing conditions. A new test to map the actual positions and orientations of each of the mirror segments will use an upgraded version of the ESPI to monitor the mirror segments after they have been mounted on the backplane of the telescope. This test will validate that the actuators controlling the position of each mirror segment can be controlled sufficiently to align all the segments to create a single optical surface.

This test will require adjusting the mirror positions, then taking up to a thousand images a second for a short period of time to verify the newly updated positions. Such a test can easily generate $10 \mathrm{~Tb}$ of imaging data in only 7 minutes. The Python software we developed for previous ESPI tests will be used as the basis for the data acquisition and data processing systems for this new test, including synthesizing data from additional measuring devices. The only way to keep up with this test will be to use multiple systems processing data in parallel to process the data quickly enough to allow the test to proceed as needed, much as we did with the reprocessing of the original ESPI data. In short, Python's rapid development capabilities, fast array handling, and ability to run the same code on multiple systems in parallel will be critical to the success of this new test. 